\title{
Existence of Pure Strategy Nash Equilibrium in Bertrand-Edgeworth Oligopolies
}

\author{
Attila Tasnádi \\ Department of Mathematics, Budapest University of Economic Sciences, \\ H-1093 Budapest, Fővám tér 8, Hungary* \\ February 3, 1999. \\ Appeared in Economics Letters 63(1999), p. 201-206, CElsevier Science \\ S.A.

\begin{abstract}
This article is searching for necessary and sufficient conditions which are to be imposed on the demand curve to guarantee the existence of pure strategy Nash equilibrium in a Bertrand-Edgeworth game with capacity constraints.
\end{abstract}

Keywords: Duopoly; Oligopoly.

JEL classification: D43; L13.

\section{Introduction}

We will investigate Bertrand-Edgeworth oligopoly with capacity constraints. We assume that the oligopolists' products are homogeneous. Furthermore we assume that there is no advertising, no possibility of outside entry into the market, and that the oligopolists possess complete information. In the Bertrand-Edgeworth game quantities and prices are both decision variables.

For a full specification of the model we need a so-called rationing rule. The aggregate demand function and the rationing rule together contain enough information on the determination of the sales of the oligopolists. We will only consider the two most frequently used rationing rules in the literature: the efficient and the proportional rationing rules. For a description of these rationing rules see for example Tirole (1988).

It has been shown for linear demand curves that when capacities are either small or large, then the Bertrand-Edgeworth duopoly with capacity constraints has an equilibrium in pure strategies (see Tirole (1988) or Wolfstetter (1993)). However, for capacities in an intermediate range, the model only has an equilibrium in mixed strategies. The mixed strategy equilibrium was

\footnotetext{
*Telephone: (+36 1) 4566782, E-mail: attila.tasnadi@math.bke.hu
} 
computed in closed form by Beckman (1965) for proportional rationing and by Levitan and Shubik (1972) for efficient rationing. Dasgupta and Maskin (1986) demonstrated the existence of mixed strategy equilibrium in the case of proportional rationing for demand curves which intersect both axes.

In section 2 we will show that if we impose assumptions on the elasticity of the demand curve, then pure strategy equilibrium will exist at all capacity levels in a Bertrand-Edgeworth duopoly. So for a certain class of demand curves a nondegenerate mixed strategy equilibrium will never arise.

In section 3 we will consider the oligopolistic case. We will show that as the number of firms increases, the Nash equilibrium price approaches the oligopolists' marginal costs. Similar convergence results have been obtained by Vives (1986) for efficient rationing and by Allen and Hellwig (1986) for proportional rationing. Due to the assumptions imposed on the demand curve our proof will be very simple.

\section{Duopoly}

First we need to specify the class of demand functions we will investigate.

Assumption 2.1 $\forall p>0: D(p)>0$ and $D^{\prime}(p)<0$.

We denote by $\epsilon(p)$ the price elasticity of the demand curve. Regarding the oligopolists we make the following assumptions:

Assumption 2.2 There are $N$ oligopolists on the market with zero marginal costs and $0<k_{i}$ capacity constraints $(i \in[1 . . N])$. Each of them can set his price $\left(p_{i}\right)$ and quantity $\left(q_{i}\right)$ simultaneously.

In this section we will only consider duopolies.

The following proposition formulates a necessary and sufficient condition, which has to be imposed on the demand curve to guarantee the existence of pure strategy equilibrium in the case of efficient rationing.

Proposition 2.3 Under the assumptions of 2.1, 2.2 and efficient rationing we can formulate the statements below about the corresponding BertrandEdgeworth duopoly game:

1. If

$$
\forall p>0: \epsilon(p) \leq-1,
$$

then there exists a unique pure strategy Nash equilibrium for all $k_{1}>0$ and $k_{2}>0$. The equilibrium is given by

$$
q_{i}^{*}=k_{i} \quad \text { and } \quad p_{1}^{*}=p_{2}^{*}=D^{-1}\left(k_{1}+k_{2}\right) .
$$


2. If $D^{\prime}$ is continuous and $\exists p>0: \epsilon(p)>-1$ then there are positive $k_{1}$ and $k_{2}$ capacity constraints, such that pure strategy Nash equilibrium does not exist.

Proof: 1. First we check that (1) implies $\lim _{p \rightarrow 0} D(p)=\infty$. Assume not; then $\lim _{p \rightarrow 0} D(p)<\infty$ because $D$ is decreasing, and so $\lim _{p \rightarrow 0} p D(p)=0$ would follow. From (1) we obtain, that $p D(p)$ is nonincreasing on $(0, \infty)$. Hence we get $\forall p>0: p D(p) \leq 0$, which contradicts the obviously true $\forall p>0: p D(p)>0$ statement. So we can conclude that a demand curve satisfying (1) does not cut the horizontal axis. Hence, $D^{-1}\left(k_{1}+k_{2}\right)$ is well defined and $p_{i}^{*}>0$.

Now we will show that only (2) can be an equilibrium. No equilibrium can exist with $p_{1}<p_{2}$ because, if $D\left(p_{1}\right)>k_{1}$, firm 1 will want to increase its price, and if $D\left(p_{1}\right) \leq k_{1}$, firm 2 will wish to reduce its price below $p_{2}$. Similarly, no equilibrium is possible with $p_{2}>p_{1}$. There cannot be an equilibrium with $p_{1}=p_{2}>p_{i}^{*}$, since both firms have the incentive to lower their prices slightly. It is obvious that a price below $p_{i}^{*}$ cannot be rational for any firm. Hence, we can rule out prices below $p_{i}^{*}$.

Finally, we have to show that raising prices unilaterally above $p_{i}^{*}$ will not increase firm $i$ 's profit. We will show this for firm 1 . Therefore we can establish that the residual profit function for firm 1 using the residual demand function does not increase in price. Under efficient rationing the residual profit function is: $\pi^{r}(p)=p D^{r}(p)=p\left(D(p)-k_{2}\right)$ for $p>p_{2}^{*}$. The nonpositivity of the first derivative is a sufficient condition, or formally

$$
\frac{\mathrm{d} \pi^{r}}{\mathrm{~d} p}(p)=p D^{\prime}(p)+D(p)-k_{2} \leq 0 \quad \Leftrightarrow \quad \epsilon(p) \leq-1+\frac{k_{2}}{D(p)} .
$$

This inequality is satisfied because of assumption (1).

2. Define the function $F(p):=p D^{\prime}(p)+D(p)$. We can pick an open interval $I=(a, b)$ from $F^{-1}((0, \infty))$ and fix any $\tilde{p} \in I$. Obviously we can choose a capacity $0<k_{1}<D(b)$ for firm 1 such that $F(\tilde{p})-k_{1}>0$. We can verify that $D(\tilde{p})>k_{1}$ also holds. So we can set the capacity for firm 2 as $k_{2}=D(\tilde{p})-k_{1}$.

Reasoning similar to that in point 1 shows that only $p_{i}:=\tilde{p}$ could be an equilibrium price. But in the case of $p_{i}=\tilde{p}$ firm 2 has an incentive to raise its price because $\tilde{p} D^{\prime}(\tilde{p})+D(\tilde{p})-k_{1}=F(\tilde{p})>0$.

For example the demand function $D(p)=p^{-\frac{1}{\alpha}}$, where $p \geq 0$ and $0<$ $\alpha \leq 1$, satisfies the assumptions of point 1 of proposition 2.3. So for these the Bertrand-Edgeworth game will have a pure strategy equilibrium.

Assumption 2.1 can be replaced in proposition 2.3 with $\forall \bar{p}>p>0$ : $D(p)>0, D^{\prime}(p)<0$ and $\forall p \geq \bar{p}: D(p)=0$. We have only to consider that 
both firms will set a price below $\bar{p}$. We can check that if $\lim _{p \rightarrow \bar{p}-0} D^{\prime}(p)$ is bounded, then $\lim _{p \rightarrow \bar{p}-0} \epsilon(p)=-\infty$. Hence, there are demand curves that are price elastic at all price levels and cut the vertical axis. As we already showed in the proof of proposition 2.3 they cannot cut the horizontal axis.

Dasgupta and Maskin (1986) proved in their third annotation that in case of random rationing a pure strategy Nash equilibrium may not exist, if the demand function is price inelastic at the $D^{-1}\left(k_{1}+k_{2}\right)$ price. So an identical proposition to 2.3 holds for proportional rationing. This can be demonstrated as proposition 2.3. One difference is that we have to use the

$$
\pi^{r}(p)=p D^{r}(p)=p D(p)\left(1-\frac{k_{2}}{D\left(p_{2}^{*}\right)}\right)
$$

residual profit function. The other difference is that the nonexistence part can be shown more easily. Particularly for $k_{1}=k_{2}=D(\tilde{p}) / 2$ capacity constraints, there does not exist a pure strategy equilibrium. Allen and Hellwig (1986) gave also in their proposition 3.1 a necessary and sufficient condition for the existence of pure strategy equilibrium for proportional rationing.

Considering the proof of proposition 2.3 we can recognize that the selection of such capacity levels for which the Bertrand-Edgeworth game has no pure strategy equilibria can require the selection of a very small capacity constraint for firm 1. Therefore we can say more in the case of efficient rationing.

Proposition 2.4 Under the assumptions of 2.1, 2.2, efficient rationing and assuming that the set of admissible capacities is

$$
\mathrm{K}_{\alpha}:=\left\{\left(k_{1}, k_{2}\right) \in \mathbf{R}^{2} \mid k_{i}>0, \frac{k_{i}}{k_{1}+k_{2}} \geq \alpha, i=1,2\right\}
$$

for some $0<\alpha \leq \frac{1}{2}$, we can make the following statements about the corresponding Bertrand-Edgeworth duopoly game:

1. If

$$
\forall p>0: \epsilon(p) \leq-1+\alpha
$$

then there exists a unique pure strategy Nash equilibrium for all $\left(k_{1}, k_{2}\right) \in \mathrm{K}_{\alpha}$. The equilibrium is given by (2).

2. If $D^{\prime}$ is continuous and

$$
\exists p>0: \epsilon(p)>-1+\alpha
$$

then there are $\left(k_{1}, k_{2}\right) \in \mathrm{K}_{\alpha}$ so that pure strategy equilibrium does not exist. 
Proof: 1 . As in the proof of proposition 2.3 we have to show that (6) implies $\lim _{p \rightarrow 0} D(p)=\infty$. First we have to prove that $\lim _{p \rightarrow 0} D^{r}(p)=\infty$. By using now the residual demand function we can do this similarly to the proof of proposition 2.3. From that $\lim _{p \rightarrow 0} D(p)=\infty$ follows immediately.

As we have already seen in the proof of proposition 2.3 the only candidate for an equilibrium price is $p^{*}:=p_{1}^{*}=p_{2}^{*}$. We have to show that raising prices unilaterally above $p_{i}^{*}$ does not increase firm $i$ 's profit. We will prove this for firm 1. Again, condition (3) has to be verified. But (3) is satisfied, because we have for all $p>p_{2}^{*}: D(p)<k_{1}+k_{2}$ and in consideration of our assumption (6)

$$
\epsilon(p) \leq-1+\alpha \leq-1+\frac{k_{2}}{k_{1}+k_{2}}<-1+\frac{k_{2}}{D(p)}
$$

holds for all $\left(k_{1}, k_{2}\right) \in \mathrm{K}_{\alpha}$. So we can conclude that it is not worthwhile for firm 1 to set its price above $p_{1}^{*}$.

2. Define the function $G(p):=p D^{\prime}(p)+(1-\alpha) D(p) . G$ is continuous. Therefore, we can pick an open interval $I=(a, b)$ from $G^{-1}((0, \infty))$, because we assumed (7). Fix any $\tilde{p} \in I$. Let $k_{1}:=\alpha D(\tilde{p})$ and $k_{2}:=(1-\alpha) D(\tilde{p})$. It is obvious that $\left(k_{1}, k_{2}\right) \in \mathrm{K}_{\alpha}$ and $D(b)<k_{1}+k_{2}<D(a)$. But now in the case of $p_{1}=p_{2}=\tilde{p}$ firm 2 has an incentive to raise its price because $\tilde{p} D^{\prime}(\tilde{p})+D(\tilde{p})-k_{1}=G(\tilde{p})>0$.

Restricting the capacities to $K_{\alpha}$ implies that one firm's capacity could not be arbitrarily small relative to the other firm's capacity. This restriction is quite acceptable for some $\alpha$ because if we want to model a duopoly, then we essentially will not be interested in a market in which one firm is relatively negligible with respect to the other firm.

The result of proposition 2.4 is that as long as the size of both firms is significant relative to each other, we can assure the existence of pure strategy equilibrium even if the demand curve has price elastic parts. This result is considerable because we now know that there are demand curves with price elastic parts and with corresponding ranges of capacities for which the Bertrand-Edgeworth game possesses equilibrium in pure strategies, such that even a monopoly without capacity constraint has a profitmaximizing price. Particularly when $\alpha=\frac{1}{2}$ the two firms have the same capacity.

One must also be aware that efficient rationing cannot be replaced by proportional rationing in proposition 2.4.

\section{Oligopoly}

We can state analogous propositions to those in section 2 for oligopolies. 
Proposition 3.1 Under the assumptions of 2.1, 2.2, the continuity of $D^{\prime}$ and efficient or proportional rationing the Bertrand-Edgeworth oligopoly game has a unique pure strategy Nash equilibrium for all $k_{i}>0$ capacities, if and only if the demand curve satisfies (1). If (1) holds, then the equilibrium is $\forall i \in[1 . . N]$ :

$$
q_{i}^{*}=k_{i} \quad \text { and } \quad p_{i}^{*}=D^{-1}\left(\sum_{i=1}^{N} k_{i}\right) .
$$

Proof: The proof of sufficiency can be done similarly to the proof in proposition 2.3. We have to prove that if the firms' prices are not all identical, then we cannot have a pure strategy equilibrium. Furthermore we have to show that (9) is an equilibrium.

In order to prove the necessity we can choose for the first $N-1$ firms capacity constraints such that $F(\tilde{p})-\sum_{i=1}^{N-1} k_{i}>0$. Now we can set the capacity for firm $N$ as $k_{N}=D(\tilde{p})-\sum_{i=1}^{N-1} k_{i}$. It can be verified that firm $N$ has an incentive to raise its price.

Corollary 3.2 If the aggregate capacity $\sum_{i} k_{i}$ tends to infinity as we increase the number of oligopolists to infinity, then the equilibrium price approaches zero, which is assumed to equal the oligopolists' marginal costs.

Similar results have been obtained by Vives (1986) for efficient rationing and by Allen and Hellwig (1986) for proportional rationing. Our proof was very simple because due to our assumptions imposed on the demand curve we did not have to deal with mixed strategy equilibria.

According to proposition 2.4 we can state more in the case of efficient rationing and equal capacities.

Proposition 3.3 Under the assumptions of 2.1, 2.2, the continuity of $D^{\prime}$, efficient rationing, and equal capacities $(k)$ our Bertrand-Edgeworth oligopoly game has a unique pure strategy Nash equilibrium for all $k>0$, if and only if

$$
\forall p>0: \epsilon(p) \leq-1+\frac{1}{N}
$$

If (10) holds, then the equilibrium is given by $\forall i \in[1 . . N]$ :

$$
q_{i}^{*}=k \quad \text { and } \quad p_{i}^{*}=D^{-1}(N k) .
$$

Proof: The proof is analogous to that of proposition 2.4 .

To guarantee the existence of a pure strategy Nash equilibrium at all capacity levels under the assumptions of proposition 3.3, we need not assume a price elastic demand curve. But the more oligopolists we have, the less demand curves secure equilibrium at all capacity levels. 


\section{Conclusions}

We have shown that for a special class of demand functions the lack of pure strategy equilibrium does not arise in the Bertrand-Edgeworth game with capacity constraints. Furthermore for demand functions outside of this class there always can be found capacity constraints, such that pure strategy equilibrium does not exist. However demand functions in this special class do not intersect the horizontal axis. For efficient rationing and equal capacities they still can be price elastic.

\section{Acknowledgements}

I am grateful to Péter Tallos and an anonymous referee for comments and suggestions. I also wish to thank Ferenc Szidarovszky for helpful remarks.

\section{References}

Allen, B. and M. Hellwig, 1986, Bertrand-Edgeworth oligopoly in large markets, Review of Economic Studies 53, 175-204.

Beckmann, M.J., with the assistance of D. Hochstädter, 1965, BertrandEdgeworth duopoly revisited, in Rudolf Henn, ed., Operations ResearchVerfahren, Vol. III, (Hain, Meisenheim) 55-68.

Dasgupta, P. and E. Maskin, 1986, The existence of equilibria in discontinuous games, II: Applications, Review of Economic Studies 53, 27-41.

Levitan, R. and M. Shubik, 1972, Price duopoly and capacity constraints, International Economic Review 13, 111-122.

Shubik, M., 1955, A comparison of treatments of a duopoly problem, Part II, Econometrica 23, 417-431.

Tirole, J., 1988, The Theory of Industrial Organization (Massachusetts Institute of Technology, Cambridge).

Vives, X., 1986, Rationing rules and Bertrand-Edgeworth equilibria in large markets, Economics Letters 21, 113-116.

Wolfstetter, E., 1993, Oligopoly and Industrial Organization, Discussion Paper no. 10, Economics Series, (Humboldt University, Berlin). 